\title{
Integrin, Exosome and Kidney Disease
}

\author{
An-Ran Shen, Xin Zhong, Tao-Tao Tang, Cui Wang, Jing Jing, Bi-Cheng Liu and Lin-Li Lv* \\ Institute of Nephrology, Zhongda Hospital, Southeast University School of Medicine, Nanjing, China
}

Integrins are transmembrane receptors that function as noncovalent heterodimers that mediate cellular adhesion and migration, cell to cell communication, and intracellular signaling activation. In kidney, latency associated peptide-transforming growth factor $\beta$ (TGF- $\beta$ ) and soluble urokinase plasminogen activator receptor (suPAR) were found as the novel ligands of integrins that contribute to renal interstitial fibrosis and focal segmental glomerular sclerosis glomerulosclerosis (FSGS). Interestingly, recent studies revealed that integrins are the compositional cargo of exosomes. Increasing evidence suggested that exosomal integrin played critical roles in diverse pathophysiologic conditions such as tumor metastasis, neurological disorders, immunology regulation, and other processes. This review will focus on the biology and function of exosomal integrin, emphasizing its

OPEN ACCESS

Edited by:

Haiyong Chen,

The University of Hong Kong,

Hong Kong

Reviewed by:

Changli Wei,

Rush University, United States

Shougang Zhuang,

Brown University, United States

*Correspondence:

Lin-Li LV

Ivlinli@seu.edu.cn

Specialty section:

This article was submitted to Renal and Epithelial Physiology, a section of the journal

Frontiers in Physiology

Received: 10 November 2020

Accepted: 30 December 2020

Published: 25 January 2021

Citation:

Shen A-R, Zhong $X$, Tang $T-T$, Wang $C$, Jing J, Liu B-C and Lv L-L (2021) Integrin, Exosome and Kidney

Disease.

Front. Physiol. 11:627800.

doi: 10.3389/fphys.2020.627800 potential role in kidney disease as well as its implications in developing novel therapeutic and diagnosis approaches for kidney disease.

Keywords: exosome, integrin, extracellular vesicle, kidney disease-diagnosis, kidney disease-therapy

\section{INTRODUCTION}

Integrins are transmembrane receptors that function as noncovalent heterodimers. There are 24 distinct integrin receptors that can recognize and bind to multiple ligands such as extracellular matrix (ECM) proteins, thereby mediating cell adhesion and intracellular signaling (MorenoLayseca et al., 2019). Other novel ligands include latency associated peptide-transforming growth factor $\beta$ (L-TGF- $\beta$ ) and soluble urokinase plasminogen activator receptor (suPAR) were found to bind to integrin and participated in the pathogenesis of kidney disease. Moreover, activated integrins on diverse kidney cells in pathological conditions participated in macrophage and fibroblast activation which played important roles in diverse kidney diseases.

Exosomes are small extracellular vesicles (EVs) secreted by nearly all types of cells which are originally considered to be the garbage bins of cells to excrete unwanted materials (Johnstone et al., 1987). Recently, accumulating studies have demonstrated that exosomes participated in crosstalk between cells and also mediated communications between organs. Moreover, exosomes can serve as vectors of therapeutics and facilitate disease diagnosis in a noninvasive way (Kalluri and LeBleu, 2020). Interestingly, integrins are revealed as the important compositional components of exosomes which take responsibility for those novel functions of exosomes.

The diverse pathophysiological roles of exosomal integrins varied from guiding the homing of exosomes (Hoshino et al., 2015), signal transmission (Kalappurakkal et al., 2019), causing phenotype transition of recipient cells (Lu et al., 2018) to cell adhesion (Genschmer et al., 2019) and migration (Sung et al., 2015). Although studies have revealed essential roles of exosomal integrin in oncology, neurology, and immunology, its role in kidney pathophysiology 
remains unclear. Thus, exploring the role of exosomal integrin in kidney disease would be helpful in understanding the mechanism of kidney disease and identifying novel diagnosis and treatment strategies. Here, we review the biology and functions of integrin as well as integrin carried by exosomes. Pathophysiologic roles of exosomal integrin in diverse diseases are also discussed, especially the role and potential applications in therapy and diagnosis of kidney diseases.

\section{BIOLOGY AND FUNCTION OF INTEGRIN}

\section{Structure and Endocytic Trafficking of Integrin}

Integrins are transmembrane heterodimers which express conservatively in almost all cell types. Integrin family was initially discovered on immune cells and mediates leukocyte extravasation by binding to intercellular cell adhesion molecule-1 (ICAM-1) on vascular endothelial cells (capturing intravenous immune cells) (Dustin, 2019). Integrins display three distinct conformations (bent, extended close, and extended open), while the activity is usually observed in the extended conformation (Campbell et al., 2020). Integrins can be categorized into 24 subtypes formed by 18 types of $\alpha$ subunits and eight types of $\beta$ subunits. Among them, integrin $\alpha \mathrm{v}, \alpha 6$, and $\beta 1$ are known for pairing with diverse subunits (Moreno-Layseca et al., 2019). Each integrin subunit contains a large extracellular domain, single-time transmembrane domain, and often rather short cytoplasmic domain (Humphries et al., 2006). Studies have shown that certain integrin subtypes are expressed on specific tissue or cell or bind to certain types of cells. For example, integrin $\beta 6$ is expressed in a few subset of epithelial cells (Breuss et al., 1993). Integrin $\alpha 6 \beta 1$ and integrin $\alpha 6 \beta 4$ targeted to lung fibroblast while integrin $\alpha v \beta 5$ targeted liver Kupffer cell (Hoshino et al., 2015). However, the dominant subtype and the abundance of integrin in a specific cell type could change under certain injury conditions. For example, the dominant integrin subtype in podocyte is $\alpha 3 \beta 1$ (Kreidberg et al., 1996), while it changes into $\alpha v \beta 3$ under focal segmental glomerulosclerosis (FSGS) situation (Hayek et al., 2017).

Although the expression levels of integrin are quite stable in certain cells and tissues, they are continuously trafficking from cytoplasm to surface membrane by diverse complex pathways including the Rab family of small GTPase (Moreno-Layseca et al., 2019). This process includes integrin endocytosis into early endosome which then traffic to late endosome and recycle to the cell surface, or alternatively transport to multivesicular bodys (MVBs) and subsequent lysosome for degradation (Rainero and Norman, 2013). Generally, majority of endocytic integrins travel back to the cell surface while small fractions target to degradation (De Franceschi et al., 2015). Interestingly, integrin endocytic trafficking process shares a common intracellular structure, MVBs, with exosome (Rainero and Norman, 2013), thus integrins could also be transported via exosome which has been demonstrated in recent studies. A study using gene ontology (GO) and Kyoto Encyclopedia of Genes and Genomes (KEGG) pathway analyses also revealed a correlation between integrin signaling and exosome secretion (Zhang et al., 2020).

\section{Ligands of Integrin}

Tremendous efforts have been invested in integrin ligand discovery, the well-known integrin ligands belong to ECM proteins, newly identified ligands include L-TGF- $\beta$ compound and suPAR (Humphries et al., 2006; Campbell et al., 2020; Hayek et al., 2020). According to the binding motif on the ligand, integrins can be classified into five types, among which, the most common type is RGD-binding integrins which belong to $\alpha \mathrm{v}$ integrins. Thus, studies have been using RGD peptide to inhibit integrin $\alpha \mathrm{v}$ subtype binding (Hoshino et al., 2015). Although multiple ligands have been discovered, most of which are non-specific ligands that can bind to more than one types of integrins and mediate cell-cell adhesion in integrin A-ECM-integrin B format (Sung et al., 2015). This suggests that in studying of integrin function under certain conditions, not only specific ligands but also the existence of other integrin subtypes should be considered.

\section{Biological Functions of Integrins}

Integrins bare different biological functions according to their diverse localizations throughout the body including cellular adhesion and migration, regulation of cellular phenotypes, cell to cell communication, and intracellular signaling activation (Table 1).

Integrins were first reported as adhesion molecule in the immune system (Springer, 1990), which represented the basic function of integrins. Many studies have revealed that integrins mediated adhesion between cells or cell to ECM. In kidney, tubular epithelial cells bind to each other on the lateral surface through integrins and bind to ECM on the basal surface by integrins as well (Glynne et al., 2001). Integrins and ECM interaction is also important for cells that underwent polarization during differentiation. Studies have showed that integrin $\beta 1$ connects ECM and cytoskeletal protein on one side of the cell, which then forms the basal membrane of cells during polarization, such as epithelial cells and endothelial cells (Lee and Streuli, 2014; Moreno-Layseca et al., 2019). Besides, dysregulation of integrin or redistribution have a great impact on cellular apical and basal polarization under injury or cancerization (Glynne et al., 2001; Liu et al., 2018a).

Moreover, integrins are associated with certain cellular phenotype and function under pathological conditions. In contrast to associated nephropathy, integrin $\alpha v \beta 6$ increased in injured tubular cells, while remains at low baseline level in normal tubules. Interestingly, injured tubular cells with high integrin were able to bind with suPAR which caused further damage (Hayek et al., 2020). Integrins also participate in regulation of cell cycle in numerous pathways as determined by various in vitro and in vivo studies. Integrin $\beta 1, \beta 3$, and other subtypes have been well demonstrated to be involved in cellular proliferation (Panchatcharam et al., 2010; MorenoLayseca and Streuli, 2014; Raven et al., 2017). Integrins mediate the local niche signal which forms spatial checkpoints that enable cells progress into $S$ phase to proliferate. Reversely, some integrin subtypes or isoforms could prevent cells from progressing into cell cycle, thus inhibiting proliferation, such as integrin $\alpha 6 \mathrm{~B} \beta 6$ in colon cancer cells (Dydensborg et al., 2009).

Ligand-integrin binding leads to signaling activation intracellularly, including focal adhesion kinase (FAK), RhoA 
TABLE 1 | Expression and function of major integrin subtypes.

\begin{tabular}{|c|c|c|c|}
\hline Integrin subtype & Expression & Function & Reference \\
\hline$\alpha \vee \beta 3$ & $\begin{array}{l}\text { podocytes, endothelial, and cancer stem } \\
\text { cells }\end{array}$ & $\begin{array}{l}\text { Bind to suPAR, promote FSGS, cancer } \\
\text { progression }\end{array}$ & Hayek et al., 2017; Nieberler et al., 2017 \\
\hline$\alpha \vee \beta 6$ & epithelial cells and tumor cells & $\begin{array}{l}\text { Activate TGF- } \beta 1 \text {, tumor progression, and } \\
\text { metastasis }\end{array}$ & Breuss et al., 1993; Nieberler et al., 2017 \\
\hline$\alpha \vee \beta 8$ & $\begin{array}{l}\text { kidney glomerular mesangial cells, brain, and } \\
\text { placenta }\end{array}$ & $\begin{array}{l}\text { Activate TGF- } \beta 1 \text {, inhibit cell growth, } \\
\text { spreading, and focal contact formation }\end{array}$ & Cambier et al., 2000; Campbell et al., 2020 \\
\hline$\beta 1$ & multiple cell types & $\begin{array}{l}\text { Cell adhesion, maintain cell polarity, regulate } \\
\text { cell proliferation, and cell cycle }\end{array}$ & Liu et al., 2018a; Kormann et al., 2020 \\
\hline$\alpha 3 \beta 1$ & $\begin{array}{l}\text { kidney tubular epithelial cells, glomerular } \\
\text { endothelial cells, and podocytes }\end{array}$ & Kidney development and cell anchorage & Kreidberg et al., 1996; Glynne et al., 2001 \\
\hline$\alpha 4 \beta 1$ & reticulocytes & Blood vascular related disease progression & Rieu et al., 2000 \\
\hline$\alpha 5 \beta 1$ & endothelial cells and cancer stem cells & $\begin{array}{l}\text { Vascular morphogenesis, cancer, and } \\
\text { metastasis }\end{array}$ & Zovein et al., 2010; Nieberler et al., 2017 \\
\hline$\alpha 6 \beta 1$ & ureteric bud & $\begin{array}{l}\text { Maintains the structural integrity of the } \\
\text { kidney collecting system }\end{array}$ & Viquez et al., 2017 \\
\hline$\alpha 6 \beta 4$ & cancer cells and epithelial cells & Lung organotropic metastasis & Hoshino et al., 2015 \\
\hline
\end{tabular}

signaling, and Glycosylphosphatidylinositol-anchored proteins (GPI-APs) nanoclustering (Kalappurakkal et al., 2019). These signals can then activate downstream processes. For example, integrin $\alpha v \beta 3$ can regulate angiogenesis (Danhier et al., 2012) by promoting HIF- $1 \alpha$ expression and subsequent endothelialmesenchymal transition (EndoMT; Fan et al., 2018). Importantly, recent studies suggested that the immobilization of integrinbinding ligand and integrin conformation was essential to integrin activation (Kalappurakkal et al., 2019; Campbell et al., 2020).

\section{INTEGRINS AS COMPOSITIONAL CARGO OF EXOSOMES}

Exosomes belong to small EVs with the size of less than $200 \mathrm{~nm}$. It is excreted into extracellular space and can transfer mRNA, miRNA, lipid, and protein to receptor cells, therefore, mediating crosstalk with neighbor and remote cells (Valadi et al., 2007; Paolicelli et al., 2019; Lv et al., 2020). Exosomes share most of the compositions from the parent cells with certain cargoes selectively sorting into the vesicles. Multiple proteomic studies on exosomes have revealed that proteins that usually present include both membranous protein and luminal protein (van Niel et al., 2018). Notably, integrins are the commonly identified exosome related proteins sorted from the parent cell. Integrin can be transported by exosomes in tumor (Quaglia et al., 2020), the central nervous system (Zhang et al., 2020) or the immune system (Genschmer et al., 2019). As the common compositional cargo of exosomes, integrins were identified as one of the critical functional cargoes of exosomes in different pathophysiological conditions.

\section{PATHOPHYSIOLOGIC ROLES OF INTEGRINS TRANSPORTED BY EXOSOMES}

Exosomal integrin is a versatile form that functions actively in different pathophysiological conditions (Table 2) which attributed to guiding the homing of exosomes, signal transmission, phenotype transition of the recipient cells, and cell adhesion and migration (Figure 1). Here, the roles of exosomal integrins in tumor, neurological disorders, immunology, and other diseases were discussed.

\section{Tumor Formation, Progression and Metastasis}

In recent years, the role of exosomes in mediating tumor formation and progression has been well studied (Li et al., 2019), integrins are among the important cargoes contributing to the process. It is reported that integrin $\alpha v \beta 3$ containing small EVs from prostate cancer cells was transferred to recipient cancer cells which induced aggressive phenotype changes (Quaglia et al., 2020). Cancer cell migration was proved to be mediated by autocrine secretion of exosomes. Fibrosarcoma cells-derived exosomal integrins and fibronectin forms adhesion assembly that mediated adhesion between cell and ECM, therefore, promoted cell motility with specific directions (Sung et al., 2015). Fibronectin was coated on exosome through binding with exosomal integrin, which then anchor to cell membrane on one side and ECM on the other and contributed to cellular adhesion (Sung et al., 2015). Moreover, it is demonstrated that exosomes transfer integrin $\alpha v \beta 6$ from cancer cells to monocytes, which promoted M2 monocyte polarization and prostate cancer progression (Lu et al., 2018).

Besides, integrins on tumor-derived exosomes can determine organotropic metastasis by forming tumor microenvironment (TME) in specific organ tissues as they uptake the exosomes (Hoshino et al., 2015). They demonstrated the specific integrin subtype directed organotropic metastasis for the first time, such as integrins $\alpha 6 \beta 4$ and $\alpha 6 \beta 1$ mediated lung metastasis, while integrin $\alpha v \beta 5$ mediated liver metastasis (Hoshino et al., 2015). The targeting properties of exosomal integrin were attributed to the activation of the Src-S100A4 axis (cancer associated genes) by exosomal ITG $\alpha 6 \beta 4$ in lung fibroblasts during pre-metastatic niche formation (Hoshino et al., 2015). These findings indicated the great therapeutic potential by targeting certain integrin subtype that was involved in tumor metastasis. 
TABLE 2 | Diverse functions of exosomal integrin.

\begin{tabular}{|c|c|c|}
\hline Diseases/process & Pathophysiologic role of exosomal integrin & Reference \\
\hline \multicolumn{3}{|l|}{ Oncology } \\
\hline Tumor metastasis & Organotropic metastasis and tumor microenvironment formation & Hoshino et al., 2015 \\
\hline Prostate cancer & Cell migration and induce integrin expression through uptake of exosomes & Fedele et al., 2015 \\
\hline Prostate cancer & $\begin{array}{l}\text { Macrophage polarization and transferring } \alpha \vee \beta 6 \text { integrin from cancer cells to monocytes } \\
\text { through exosomes }\end{array}$ & Lu et al., 2018 \\
\hline Prostate cancer & Determined cargo loading of exosomes which promoted cancer cell formation & Quaglia et al., 2020 \\
\hline Fibrosarcoma & Promoted cell migration & Sung et al., 2015 \\
\hline \multicolumn{3}{|l|}{ Neurology } \\
\hline Demyelination & Oligodendrocyte precursor cells proliferation & Zhang et al., 2020 \\
\hline CNS diseases & Therapeutic protein delivery, exosome uptake, and spread of viral proteins to the brain & Yuan et al., 2017 \\
\hline \multicolumn{3}{|c|}{ ( } \\
\hline COPD & Mediated exosome adhesion to extracellular matrix & Genschmer et al., 2019 \\
\hline \multicolumn{3}{|l|}{ Gut } \\
\hline lymphocyte homing & $\begin{array}{l}\text { Exosomal integrin } \alpha 4 \beta 7 \text { target high endothelial venule (HEV) endothelial cells causing } \\
\text { diminish in lymphocyte homing niche }\end{array}$ & Myint et al., 2020 \\
\hline
\end{tabular}

\section{Neurological Disorders}

Exosomal integrins participate in the development of neurological disorders and are active in multiple trans-cellular communication processes. Proteomic analysis proved that integrin $\alpha v \beta 3$ was upregulated in interleukin-1 $\beta$ (IL-1 $\beta$ ) stimulated astrocyte-derived EVs (ADEV) and significantly increased uptake of ADEV in neurons, while integrin $\alpha v \beta 3$ blocking partially suppressed this process (You et al., 2020). Exosomal integrins also contributed to the recovery of central nervous system (CNS) degenerative diseases, which was associated with the proliferation of oligodendrocyte precursor cells (OPCs; Zhang et al., 2020). Moreover, in therapeutic studies, macrophage derived exosome contained the integrin subtype lymphocyte function-associated antigen 1 (LFA-1). This facilitated macrophage derived exosome to overcome the blood-brain barrier and deliver therapeutic protein cargos specifically to treat CNS diseases (Yuan et al., 2017).

\section{Immunology Regulation and Others}

Integrin was first discovered as adhesion molecules in immune cells that mediated extravasation (Springer, 1994). Similar to forming TMV for tumor metastasis, exosomal integrins were also involved in immune cell homing (Myint et al., 2020). Study showed that integrin $\alpha 4 \beta 7$ on T cell-derived exosomes guided the exosomes homing to the intestine through binding to mucosal addressin cell adhesion molecule-1 (MAdCAM-1; Mora et al., 2003). On the other hand, integrin $\alpha 4 \beta 7$-expressing $\mathrm{T}$ cell exosomes could suppress MAdCAM-1 expression which, therefore, inhibited subsequent lymphocyte homing to the gut (Park et al., 2019).

Besides, activated polymorphonuclear leukocyte (PMN) derived exosomes were capable of targeting ECM through MAC-1 ( $\alpha$ M $\beta 2$ integrin). This caused activation of neutrophil elastase (NE) that was coated on exosomes and lead to ECM degradation (Genschmer et al., 2019). Moreover, during reticulocyte maturation, integrin $\alpha 4 \beta 1$, that expressed commonly on the surface of reticulocyte, was cleared from the reticulocyte through exosome secretion. This reduced the risk of blood circulation complications, such as sickle-cell anemia, caused by integrin $\alpha 4 \beta 1$ on reticulocytes (Rieu et al., 2000).

\section{INTEGRINS IN KIDNEY DISEASES}

\section{Integrins Expression in Renal Cells}

Studies have revealed that integrins are expressed on various types of cells in the kidney including tubular epithelial cell (TECs) (Zhu et al., 2020), fibroblast (Bon et al., 2019), and podocyte (Hayek et al., 2017).

Tubular epithelial cells are the primary cellular component of kidney which is susceptible to diverse injuries (Liu et al., 2018b). TECs express $\alpha \mathrm{v}$ and $\beta 1$ integrins under normal conditions (Bon et al., 2019), while integrin $\alpha \mathrm{v}, \beta 1$, and $\beta 6$ are the dominant subtypes with injury (Hayek et al., 2020). ITG $\beta 6$ (gene of integrin $\beta 6$ ) was rarely identified in normal TECs but rapidly increased in the format of $\alpha v \beta 6$ subtype under injury. Moreover, a study of clinic kidney biopsy concluded that integrin $\beta 6$ was elevated in the distal tubules in diverse diseased and transplanted kidney (Trevillian et al., 2004). Highly expressed $\beta 1$ integrins are known to be involved in epithelial cell polarization which traffic from basal membrane to apical membrane under injury (Glynne et al., 2001). This could result in detachment of TECs from basal ECM and impairment of polarization, which caused further injury of tubules and dysregulation of cell secretion, since integrins played a key role in delivering molecules to the right subcellular compartments (Moreno-Layseca et al., 2019).

Fibroblasts are one of the main cellular components in renal interstitial fibrosis, they can migrate to damaged site, transform into myofibroblasts, and produce ECM. Fibroblasts normally express integrin $\alpha 1, \alpha 4, \alpha 5$, and $\beta 1$ and turn into integrin $\alpha 5, \beta 1$, and $\alpha \mathrm{v}$ under fibrosis situations (Norman and Fine, 1999), among which integrin $\alpha \mathrm{v}$ was the dominant type (Bon et al., 2019). Integrin $\alpha 5$ facilitates fibroblasts migration through binding to ECM (Lobert et al., 2010). Interestingly, integrin $\alpha \mathrm{v}$ expressed by fibroblasts binds to latent-TGF- $\beta$ and stimulates subsequent tissue fibrosis (Henderson et al., 2013). This relation between integrin and fibroblasts also presents in other organs such as colon (Peng et al., 2018), skin (van Caam et al., 2020), lung, liver (Reed et al., 2015), and pancreatic duct (Cavaco et al., 2019). 


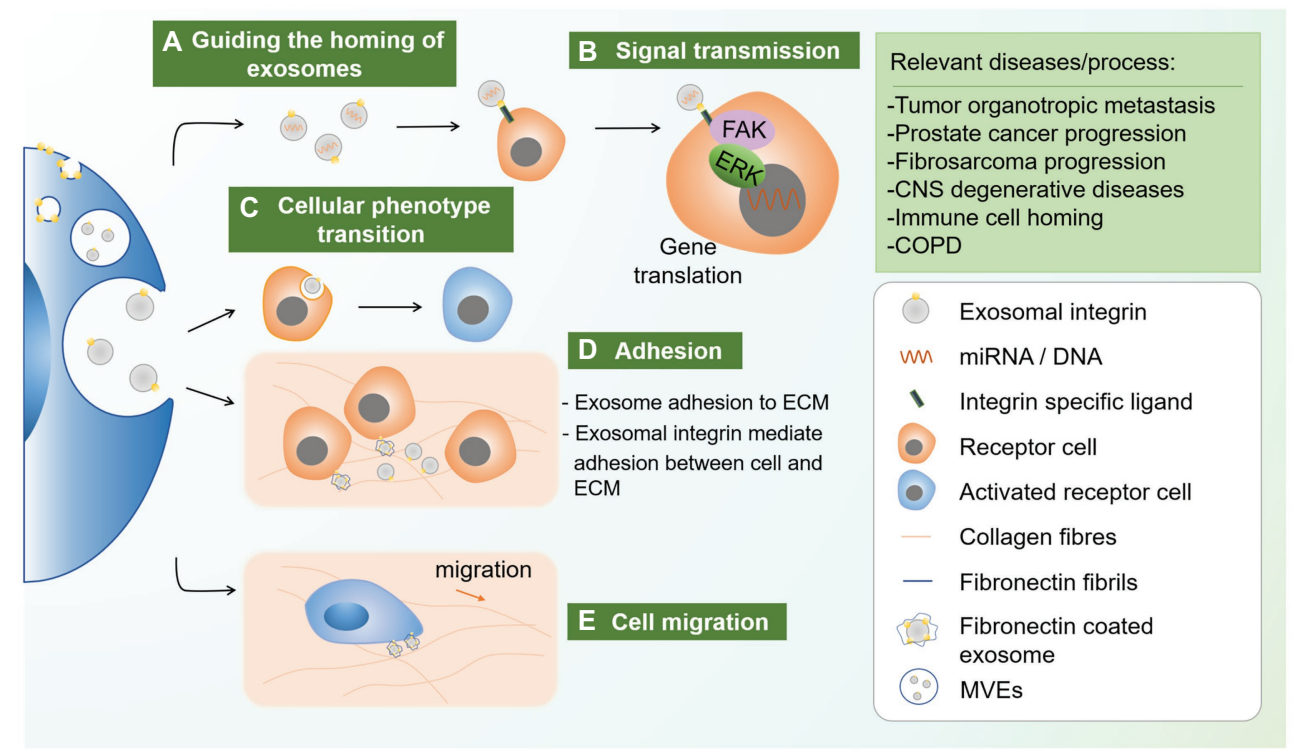

FIGURE 1 | Novel functions of exosomal integrin. Exosomal integrin played diverse roles in different disease conditions including guiding the homing of exosomes, signal transmission, cellular phenotype transition, and cellular adhesion and migration. (a) Exosome cargoes such as miRNA and protein can be delivered to neigbor and distant cells, while specific type of exosomal integrin can guide the vesicles to specific cells through integrin-ligand recognition. (b) Integrin-ligand interatction could activate intracellular signals, for example FAK and ERK-1/2, which initiate relevant gene translation. (c) Apart from intracellular signaling, endocytosis of specific exosomal integrin could also cause cellular phenotype transition of the receipient cells. (d) Exosomal integrin mediated exosome adhesion to extracellular matrix (ECM) through integrin-ECM binding. (e) Moreover, ECM, for example, fibronectin, could be coated on exosome through a process involving endocytosis of integrin $\alpha \vee \beta 1$-fibronectin complex which then sorted into MVEs. FN-coated exosomes secreted and bind to collagen fibrils, which can then coupled to cellular integrin receptors. This adhesion ensures the stable adhesion to ECM during migration. CNS, central nervous system; COPD, chronic obstructive pulmonary diseases; MVEs, multivesicular endosomes.

Podocytes are special for their foot processes and integrin $\alpha 3$ plays a critical role in its maturation. In vivo study showed that the mutation of murine integrin $\alpha 3$ gene caused abnormal kidney and lung development (Kreidberg et al., 1996). Studies reported that activated $\beta 3$ integrin on podocytes could initiate FSGS pathology in a suPAR-APOL1-integrin $\alpha v \beta 3$ tripartite complex dependent manner. The underlie mechanisms included autophagosomes formation, actin cytoskeleton dysregulation, and cell detachment (Wei et al., 2011).

\section{Novel Roles of Integrins in Kidney Disease}

Recent studies have showed that integrins bind with novel molecules and drive subsequent signaling pathways, including TGF- $\beta$ and suPAR. Distinct integrins bind with latent-TGF- $\beta$ which activates TGF- $\beta$ and downstream signals, such as Smad2/3. These signals can promote interstitial fibrosis in chronic kidney disease (CKD; Meng et al., 2016) and suppress TEC proliferation after injury in acute kidney injury (AKI; Yang et al., 2019). It was demonstrated that the increased TGF- $\beta$ signaling was initiated in the early stage of AKI which continuously expressed during recovery stage. TGF- $\beta$ expression in the tubules was companied by integrin $\beta 6$ and lead to subsequent interstitial fibrosis (Geng et al., 2012). In this regard, integrins may play a prominent role in AKI to CKD transition by activation of TGF- $\beta$.

Unlike ECM or TGF- $\beta$, suPAR is not stabilized, it is the released version of the podocyte urokinase receptor (UPAR), which function as the cellular receptor for urokinase. suPAR exists in the circulatory system and its increased concentration is associated with acute (Hayek et al., 2020) and chronic kidney injuries (Hayek et al., 2017). Several studies have reported that suPAR primarily binds with $\beta 3$ integrin on the surface of podocytes (Wei et al., 2011) by way of a tripartite complex of suPAR-APOL1 risk variants-integrin $\beta 3$ (Hayek et al., 2017). Meanwhile, suPAR bind to TECs through integrin $\beta 6$ under injured conditions and activated Racl, which bound to SRp40 at the $5^{\prime}$ end of exon 7 in versican pre-mRNA. Versican then resulted in subsequent fibroblast activation and promoted interstitial fibrosis by activating the CD44/Smad3 pathway (Han et al., 2019). Moreover, suPAR could bind to integrin $\beta 1$ and $\beta 2$ which promoted inflammation and tumor progression (Simon et al., 2000).

\section{Potential Function of Exosomal Integrins in Kidney Disease}

Studies from our group and others have demonstrated that TEC released exosomes mediated cross-talk with fibroblasts (Guan et al., 2020) and macrophages (Lv et al., 2020) which contributed to renal inflammation and fibrosis. However, the traveling direction of TEC exosomes to specific cells remains largely unknown. Since integrins are the common compositional cargoes of exosomes, it is reasonable to speculate that integrin may be critical for directing the fate of the exosomes. Indeed, 
our study showed that integrin $\alpha \mathrm{L} \beta 2$ (LFA-1) and $\alpha 4 \beta 1$ (VAL-4) on exosomes enabled them to adhere to the inflamed kidney (Tang et al., 2019). Thus, integrin on exosomes may be critical for guiding the traveling of TECs exosomes and mediated the cross-talk with specific recipient cells. Besides, due to the critical role of integrins such as integrin $\alpha \mathrm{v} \beta 6$ and $\beta 1$ on TECs, integrin carried by exosomes may play an important role in interstitial inflammation and fibrosis. Moreover, podocytes express integrin $\beta 3$ that binds with soluble particle suPAR (Wei et al., 2011), thus, podocyte may secrete exosomes with $\beta 3$ integrins and meditate cellular communication in kidney disease.

Since integrin intracellular trafficking shares multiple pathways with exosome packing and releasing, integrin may also be involve in exosome generation (Rainero and Norman, 2013). Knockdown of integrin $\beta 4$ decreased the concentration of exosomes in the cultured OPCs supernatant and the capacity to proliferate, while supplement of exosomes reversed this capacity (Zhang et al., 2020). This strongly indicated the critical role of integrin in exosome generation and function. Therefore, the role of integrins in exosomes release and cargo loading for kidney cells under pathologic conditions deserve further investigation.

Hence, as the compositional cargo, integrins carried by exosome may mediate specific cell-crosstalk which participate in the pathophysiological process of the kidney.

\section{INTEGRINS CARRIED BY EXOSOMES IN THE TARGET THERAPY AND DIAGNOSIS OF DISEASE}

Currently, exosome has been demonstrated as the promising engineered nanocarriers in therapy of disease due to its low immunogenicity, biological barrier permeability, and intrinsic targeting properties (Tang et al., 2020). Studies have showed that exosomal integrins may contribute to the properties of targeting delivery of exosomes. Integrins naturally expressed on exosomes could be used to realize target exosome therapy. Recently, it was found that macrophage-derived EV migrated toward inflamed endothelial cells which was mediated by integrin $\alpha \mathrm{L} \beta 2$ and integrin $\alpha 4 \beta 1$ on EVs in kidney (Tang et al., 2019) and brain (Yuan et al., 2017), respectively. Proteomic analysis of macrophage-derived micro vesicle (MV) carried with dexamethasone revealed that integrin $\alpha \mathrm{L} \beta 2$ (LFA-1) and $\alpha 4 \beta 1$ (VAL-4) express distinctly on the surface, which could efficiently direct MV to the inflamed kidney through recognizing ICAM-1 and vascular cell adhesion molecule-1 (VCAM-1) (Tang et al., 2019).

Since exosomal integrins are important for tumor metastasis, it might hold promise in targeted drug delivery for tumor (Qiao et al., 2020). It is demonstrated that non-small cell lung cancer cells could specifically uptake breast cancer (MDA-MB231) cell-derived exosomes (231-Exo), which was loaded with mRNA-126 that successfully inhibited lung metastasis in vivo (Nie et al., 2020). This organotropic process was depended on integrin $\beta 4$-exosome that specifically targeted surfactant protein C (SPC) on cancer cells. Moreover, EVs can be engineered to express integrin for target therapy. For example, a study used click chemistry method to conjugate integrin $\alpha v \beta 3$-specific
cRGD peptides to the surface of exosomes. The results showed the engineered exosome efficiently targeted to injured areas in the brain (Tian et al., 2018).

Based on the discovery of disease-specific integrin by various exosome proteomic studies, detection of different types of integrin carried by exosomes could be novel biomarkers of diseases. It was found that integrin is among the top 100 protein in urinary EVs proteomic studies including AKI, FSGS, autosomal dominant polycystic kidney disease (ADPKD), etc. (Merchant et al., 2017). According to proteomic analysis, integrin on urinary exosomes showed strong correlation with kidney diseases. For example, integrin signaling was identified as the most canonical represented signaling pathways correlated with inherited glomerular diseases by way of ingenuity pathway analysis (Hogan et al., 2014).

\section{CONCLUSIONS AND PERSPECTIVES}

Exosomal integrin played diverse roles in different disease conditions via mediating intercellular crosstalk. Integrins are essential for normal cellular adhesion and polarization, while specific pathogenic subtypes of integrins have the potential to trigger renal inflammation and fibrosis via activating TGF- $\beta$, epithelial-mesenchymal transition (EMT) signaling, FAK and mitogen-activated protein kinases (MAPKs). However, the role of exosomal integrin in kidney disease remains largely unknown. Exosomal integrin may contribute to the injury and repair processes of kidney disease as the novel format of integrin via mediating cellular communication and downstream signaling activation. In addition, integrins may also hold the potential to participate in intracellular exosome secretion and cargo loading which may provide a promising approach for engineering of exosome for diagnosis and therapeutic purpose.

The guiding effect of specific exosomal integrin was demonstrated in tumor or immune cells. Despite that integrin can direct the destination of exosomes, the underlie mechanism require further investigation. Nevertheless, the guiding effect of exosomal integrin provided an important pathway for developing target therapy for kidney diseases. Further investigation in the role of diverse exosomal integrin subtypes in cellular communication may allow the construction of specific targeting exosome for precise treatment of kidney disease.

\section{AUTHOR CONTRIBUTIONS}

A-RS wrote the manuscript. L-LL conceived the concept and contributed to the writing of the manuscript. All authors contributed to the literature review and approved the submitted version.

\section{FUNDING}

This study is supported by grants from National Natural Scientific Foundation of China (grant no. 31671194, 81970616, 82030024, and 81720108007) and National Key R\&D Program of China (2018YFC1314000). 


\section{REFERENCES}

Bon, H., Hales, P., Lumb, S., Holdsworth, G., Johnson, T., Qureshi, O., et al. (2019). Spontaneous extracellular matrix accumulation in a human in vitro model of renal fibrosis is mediated by alphaV integrins. Nephron 142, 328-350. doi: 10.1159/000499506

Breuss, J. M., Giuelt, N., Lu, L., Sheppard, D., and Pytela, R. (1993). Restricted distribution of integrin P6 mRNA in primate epithelial tissues. J. Histochem. Cytochem. 41, 1521-1527. doi: 10.1177/41.10.8245410

Cambier, S., Mu, D. Z., O’Connell, D., Boylen, K., Travis, W., Liu, W. H., et al. (2000). A role for the integrin alphavbeta8 in the negative regulation of epithelial cell growth. Cancer Res. 60, 7084-7093.

Campbell, M. G., Cormier, A., Ito, S., Seed, R. I., Bondesson, A. J., Lou, J., et al. (2020). Cryo-EM reveals integrin-mediated TGF-beta activation without release from latent TGF-beta. Cell 180, 490.e416-501.e416. doi: 10.1016/j.cell.2019.12.030

Cavaco, A. C. M., Rezaei, M., Caliandro, M. F., Lima, A. M., Stehling, M., Dhayat, S. A., et al. (2019). The interaction between Laminin-332 and alpha 3 beta 1 integrin determines differentiation and maintenance of CAFs, and supports invasion of pancreatic duct adenocarcinoma cells. Cancer 11:20. doi: $10.3390 /$ cancers 11010014

Danhier, F., Le Breton, A., and Preat, V. (2012). RGD-based strategies to target alpha(v) beta(3) integrin in cancer therapy and diagnosis. Mol. Pharm. 9, 2961-2973. doi: 10.1021/mp3002733

De Franceschi, N., Hamidi, H., Alanko, J., Sahgal, P., and Ivaska, J. (2015). Integrin traffic-the update. J. Cell Sci. 128, 839-852. doi: $10.1242 /$ jcs. 161653

Dustin, M. L. (2019). Integrins and their role in immune cell adhesion. Cell 177, 499-501. doi: 10.1016/j.cell.2019.03.038

Dydensborg, A. B., Teller, I. C., Groulx, J. F., Basora, N., Pare, F., Herring, E., et al. (2009). Integrin alpha $6 \mathrm{~B}$ beta 4 inhibits colon cancer cell proliferation and c-Myc activity. BMC Cancer 9:223. doi: 10.1186/1471-2407-9-223

Fan, C. S., Chen, W. S., Chen, L. L., Chen, C. C., Hsu, Y. T., Chua, K. V., et al. (2018). Osteopontin-integrin engagement induces HIF-1 alpha-TCF12mediated endothelial-mesenchymal transition to exacerbate colorectal cancer. Oncotarget 9, 4998-5015. doi: 10.18632/oncotarget.23578

Fedele, C., Singh, A., Zerlanko, B. J., Iozzo, R. V., and Languino, L. R. (2015). The $\alpha v \beta 6$ integrin is transferred intercellularly via exosomes. J. Biol. Chem. 290, 4545-4551. doi: 10.1074/jbc.C114.617662

Geng, H., Lan, R., Singha, P. K., Gilchrist, A., Weinreb, P. H., Violette, S. M., et al. (2012). Lysophosphatidic acid increases proximal tubule cell secretion of profibrotic cytokines PDGF-B and CTGF through LPA2- and Galphaqmediated rho and alphavbeta6 integrin-dependent activation of TGF-beta. Am. J. Pathol. 181, 1236-1249. doi: 10.1016/j.ajpath.2012.06.035

Genschmer, K. R., Russell, D. W., Lal, C., Szul, T., Bratcher, P. E., Noerager, B. D., et al. (2019). Activated PMN exosomes: pathogenic entities causing matrix destruction and disease in the lung. Cell 176, 113.e115-126.e115. doi: 10.1016/j.cell.2018.12.002

Glynne, P. A., Picot, J., and Evans, T. J. (2001). Coexpressed nitric oxide synthase and apical $\aleph_{1} 1$ integrins influence tubule cell adhesion after cytokine-induced injury. J. Am. Soc. Nephrol. 12, 2370-2383.

Guan, H., Peng, R., Mao, L. K., Fang, F., Xu, B., and Chen, M. (2020). Injured tubular epithelial cells activate fibroblasts to promote kidney fibrosis through miR-150-containing exosomes. Exp. Cell Res. 392:112007. doi: 10.1016/j. yexcr.2020.112007

Han, R., Hu, S., Qin, W., Shi, J., Hou, Q., Wang, X., et al. (2019). C3a and suPAR drive versican V1 expression in tubular cells of focal segmental glomerulosclerosis. JCI Insight 4:e122912. doi: 10.1172/jci.insight.122912.

Hayek, S. S., Koh, K. H., Grams, M. E., Wei, C., Ko, Y. -A., Li, J., et al. (2017). A tripartite complex of suPAR, APOL1 risk variants and $\alpha v \beta 3$ integrin on podocytes mediates chronic kidney disease. Nat. Med. 23, 945-953. doi: $10.1038 / \mathrm{nm} .4362$

Hayek, S. S., Leaf, D. E., Samman Tahhan, A., Raad, M., Sharma, S., Waikar, S. S., et al. (2020). Soluble urokinase receptor and acute kidney injury. N. Engl. J. Med. 382, 416-426. doi: 10.1056/NEJMoa1911481

Henderson, N. C., Arnold, T. D., Katamura, Y., Giacomini, M. M., Rodriguez, J. D., McCarty, J. H., et al. (2013). Targeting of alpha(v) integrin identifies a core molecular pathway that regulates fibrosis in several organs. Nat. Med. 19, 1617-1624. doi: $10.1038 / \mathrm{nm} .3282$
Hogan, M. C., Johnson, K. L., Zenka, R. M., Charlesworth, M. C., Madden, B. J., Mahoney, D. W., et al. (2014). Subfractionation, characterization, and in-depth proteomic analysis of glomerular membrane vesicles in human urine. Kidney Int. 85, 1225-1237. doi: 10.1038/ki.2013.422

Hoshino, A., Costa-Silva, B., Shen, T. L., Rodrigues, G., Hashimoto, A., Tesic Mark, M., et al. (2015). Tumour exosome integrins determine organotropic metastasis. Nature 527, 329-335. doi: 10.1038/nature15756

Humphries, J. D., Byron, A., and Humphries, M. J. (2006). Integrin ligands at a glance. J. Cell Sci. 119, 3901-3903. doi: 10.1242/jcs.03098

Johnstone, R. M., Adam, M., Hammond, J. R., Orr, L., and Turbide, C. (1987). Vesicle formation during reticulocyte maturation-association of plasmamembrane activities with released vesicles (exosomes). J. Biol. Chem. 262, 9412-9420.

Kalappurakkal, J. M., Anilkumar, A. A., Patra, C., van Zanten, T. S., Sheetz, M. P., and Mayor, S. (2019). Integrin mechano-chemical signaling generates plasma membrane nanodomains that promote cell spreading. Cell 177, 1738.e1723-1756.e1723. doi: 10.1016/j.cell.2019.04.037

Kalluri, R., and LeBleu, V. S. (2020). The biology, function, and biomedical applications of exosomes. Science 367:eaau6977. doi: 10.1126/science.aau6977

Kormann, R., Kavvadas, P., Placier, S., Vandermeersch, S., Dorison, A., Dussaule, J. C., et al. (2020). Periostin promotes cell proliferation and macrophage polarization to drive repair after AKI. J. Am. Soc. Nephrol. 31, 85-100. doi: 10.1681/ASN.2019020113

Kreidberg, J. A., Donovan, M. J., Goldstein, S. L., Rennke, H., Shepherd, K., Jones, R. C., et al. (1996). Alpha 3 beta 1 integrin has a crucial role in kidney and lung organogenesis. Development 122, 3537-3547.

Lee, J. L., and Streuli, C. H. (2014). Integrins and epithelial cell polarity. J. Cell Sci. 127, 3217-3225. doi: 10.1242/jcs.146142

Li, K. Y., Chen, Y. H., Li, A., Tan, C. L., and Liu, X. B. (2019). Exosomes play roles in sequential processes of tumor metastasis. Int. J. Cancer 144, 1486-1495. doi: $10.1002 /$ ijc. 31774

Liu, B.-C., Tang, T.-T., Lin-Li, L., and Lan, H.-Y. (2018b). Renal tubule injury: a driving force toward chronic kidney disease. Kidney Int. 93, 568-579. doi: $10.1016 /$ j.kint.2017.09.033

Liu, B. B., Zheng, X., Meng, F. F., Han, Y. W., Song, Y. W., Liu, F. F., et al. (2018a). Overexpression of beta 1 integrin contributes to polarity reversal and a poor prognosis of breast invasive micropapillary carcinoma. Oncotarget 9, 4338-4353. doi: 10.18632/oncotarget.22774

Lobert, V. H., Brech, A., Pedersen, N. M., Wesche, J., Oppelt, A., Malerod, L., et al. (2010). Ubiquitination of alpha 5 beta 1 integrin controls fibroblast migration through lysosomal degradation of fibronectin-integrin complexes. Dev. Cell 19, 148-159. doi: 10.1016/j.devcel.2010.06.010

Lu, H., Bowler, N., Harshyne, L. A., Craig Hooper, D., Krishn, S. R., Kurtoglu, S., et al. (2018). Exosomal alphavbeta6 integrin is required for monocyte M2 polarization in prostate cancer. Matrix Biol. 70, 20-35. doi: 10.1016/j. matbio.2018.03.009

Lv, L. L., Feng, Y., Wu, M., Wang, B., Li, Z. L., Zhong, X., et al. (2020). Exosomal miRNA-19b-3p of tubular epithelial cells promotes M1 macrophage activation in kidney injury. Cell Death Differ. 27, 210-226. doi: 10.1038/ s41418-019-0349-y

Meng, X. M., Nikolic-Paterson, D. J., and Lan, H. Y. (2016). TGF-beta: the master regulator of fibrosis. Nat. Rev. Nephrol. 12, 325-338. doi: 10.1038/nrneph.2016.48

Merchant, M. L., Rood, I. M., Deegens, J. K. J., and Klein, J. B. (2017). Isolation and characterization of urinary extracellular vesicles: implications for biomarker discovery. Nat. Rev. Nephrol. 13, 731-749. doi: 10.1038/nrneph.2017.148

Mora, J. R., Bono, M. R., Manjunath, N., Weninger, W., Cavanagh, L. L., Rosemblatt, M., et al. (2003). Selective imprinting of gut-homing T cells by Peyer's patch dendritic cells. Nature 424, 88-93. doi: 10.1038/nature01726

Moreno-Layseca, P., Icha, J., Hamidi, H., and Ivaska, J. (2019). Integrin trafficking in cells and tissues. Nat. Cell Biol. 21, 122-132. doi: 10.1038/s41556-018-0223-Z

Moreno-Layseca, P., and Streuli, C. H. (2014). Signalling pathways linking integrins with cell cycle progression. Matrix Biol. 34, 144-153. doi: 10.1016/j. matbio.2013.10.011

Myint, P. K., Park, E. J., Gaowa, A., Kawamoto, E., and Shimaoka, M. (2020). Targeted remodeling of breast cancer and immune cell homing niches by exosomal integrins. Diagn. Pathol. 15:38. doi: 10.1186/s13000-020-00959-3

Nie, H., Xie, X., Zhang, D., Zhou, Y., Li, B., Li, F., et al. (2020). Use of lungspecific exosomes for miRNA-126 delivery in non-small cell lung cancer. Nanoscale 12, 877-887. doi: 10.1039/c9nr09011h 
Nieberler, M., Reuning, U., Reichart, F., Notni, J., Wester, H. J., Schwaiger, M., et al. (2017). Exploring the role of RGD-recognizing integrins in cancer. Cancer 9:116. doi: 10.3390/cancers9090116

Norman, J. T., and Fine, L. G. (1999). Progressive renal disease: fibroblasts, extracellular matrix, and integrins. Exp. Nephrol. 7, 167-177. doi: 10.1159/000020597

Panchatcharam, M., Miriyala, S., Yang, F., Leitges, M., Chrzanowska-Wodnicka, M., Quilliam, L. A., et al. (2010). Enhanced proliferation and migration of vascular smooth muscle cells in response to vascular injury under hyperglycemic conditions is controlled by beta 3 integrin signaling. Int. J. Biochem. Cell Biol. 42, 965-974. doi: 10.1016/j.biocel.2010.02.009

Paolicelli, R. C., Bergamini, G., and Rajendran, L. (2019). Cell-to-cell communication by extracellular vesicles: focus on microglia. Neuroscience 405, 148-157. doi: 10.1016/j.neuroscience.2018.04.003

Park, E. J., Prajuabjinda, O., Soe, Z. Y., Darkwah, S., Appiah, M. G., Kawamoto, E., et al. (2019). Exosomal regulation of lymphocyte homing to the gut. Blood Adv. 3, 1-11. doi: 10.1182/bloodadvances.2018024877

Peng, C., Zou, X. Q., Xia, W. Y., Gao, H. J., Li, Z. Q., Liu, N. Q., et al. (2018). Integrin alpha $\mathrm{v}$ beta 6 plays a bi-directional regulation role between colon cancer cells and cancer-associated fibroblasts. Biosci. Rep. 38:12. doi: 10.1042/ bsr20180243

Qiao, L., Hu, S. Q., Huang, K., Su, T., Li, Z. H., Vandergriff, A., et al. (2020). Tumor cell-derived exosomes home to their cells of origin and can be used as Trojan horses to deliver cancer drugs. Theranostics 10, 3474-3487. doi: 10.7150/thno.39434

Quaglia, F., Krishn, S. R., Daaboul, G. G., Sarker, S., Pippa, R., Domingo-Domenech, J., et al. (2020). Small extracellular vesicles modulated by alphaVbeta3 integrin induce neuroendocrine differentiation in recipient cancer cells. J. Extracell. Vesicles 9:1761072. doi: 10.1080/20013078.2020.1761072

Rainero, E., and Norman, J. C. (2013). Late endosomal and lysosomal trafficking during integrin-mediated cell migration and invasion. BioEssays 35, 523-532. doi: 10.1002/bies.201200160

Raven, A., Lu, W. Y., Man, T. Y., Ferreira-Gonzalez, S., O’Duibhir, E., Dwyer, B. J., et al. (2017). Cholangiocytes act as facultative liver stem cells during impaired hepatocyte regeneration. Nature 547, 350-354. doi: 10.1038/nature23015

Reed, N. I., Jo, H., Chen, C., Tsujino, K., Arnold, T. D., DeGrado, W. F., et al. (2015). The alpha(v)beta(1) integrin plays a critical in vivo role in tissue fibrosis. Sci. Transl. Med. 7:288ra79. doi: 10.1126/scitranslmed.aaa5094

Rieu, S. P., Minard, C. G., Rabesandratana, H., Sainte-Marie, J., and Vidal, M. (2000). Exosomes released during reticulocyte maturation bind to fibronectin via integrin $\alpha 4 \beta 1$. Eur. J. Biochem. 267, 583-590. doi: 10.1046/j. 1432-1327.2000.01036.x

Simon, D. I., Wei, Y., Zhang, L., Rao, N. K., Xu, H., Chen, Z. P., et al. (2000). Identification of a urokinase receptor-integrin interaction site-promiscuous regulator of integrin function. J. Biol. Chem. 275, 10228-10234. doi: 10.1074/ jbc. 275.14 .10228

Springer, T. A. (1990). Adhesion receptors of the immune system. Nature 346, 425-434. doi: 10.1038/346425a0

Springer, T. A. (1994). Traffic signals for lymphocyte recirculation and leukocyte emigration: the multistep paradigm. Cell 76, 301-314. doi: 10.1016/00928674(94)90337-9

Sung, B. H., Ketova, T., Hoshino, D., Zijlstra, A., and Weaver, A. M. (2015). Directional cell movement through tissues is controlled by exosome secretion. Nat. Commun. 6:7164. doi: 10.1038/ncomms8164

Tang, T. T., Lv, L. L., Wang, B., Cao, J. Y., Feng, Y., Li, Z. L., et al. (2019). Employing macrophage-derived microvesicle for kidney-targeted delivery of dexamethasone: an efficient therapeutic strategy against renal inflammation and fibrosis. Theranostics 9, 4740-4755. doi: 10.7150/thno.33520

Tang, T. T., Wang, B., Lv, L. L., and Liu, B. C. (2020). Extracellular vesiclebased nanotherapeutics: emerging frontiers in anti-inflammatory therapy. Theranostics 10, 8111-8129. doi: 10.7150/thno.47865
Tian, T., Zhang, H. X., He, C. P., Fan, S., Zhu, Y. L., Qi, C., et al. (2018). Surface functionalized exosomes as targeted drug delivery vehicles for cerebral ischemia therapy. Biomaterials 150, 137-149. doi: 10.1016/j.biomaterials.2017.10.012

Trevillian, P., Paul, H., Millar, E., Hibberd, A., and Agrez, M. V. (2004). $\alpha v \beta 6$ integrin expression in diseased and transplanted kidneys. Kidney Int. 66, 1423-1433. doi: 10.1111/j.1523-1755.2004.00904.x

Valadi, H., Ekstrom, K., Bossios, A., Sjostrand, M., Lee, J. J., and Lotvall, J. O. (2007). Exosome-mediated transfer of mRNAs and microRNAs is a novel mechanism of genetic exchange between cells. Nat. Cell Biol. 9, 654-659. doi: $10.1038 / \mathrm{ncb} 1596$

van Caam, A., Aarts, J., van Ee, T., Vitters, E., Koenders, M., van de Loo, F., et al. (2020). TGF beta-mediated expression of TGF beta-activating integrins in SSc monocytes: disturbed activation of latent TGF beta? Arthritis Res. Ther. 22:42. doi: 10.1186/s13075-020-2130-5

van Niel, G., D’Angelo, G., and Raposo, G. (2018). Shedding light on the cell biology of extracellular vesicles. Nat. Rev. Mol. Cell Biol. 19, 213-228. doi: 10.1038/nrm.2017.125

Viquez, O. M., Yazlovitskaya, E. M., Tu, T., Mernaugh, G., Secades, P., McKee, K. K., et al. (2017). Integrin alpha6 maintains the structural integrity of the kidney collecting system. Matrix Biol. 57-58, 244-257. doi: 10.1016/j. matbio.2016.12.003

Wei, C., El Hindi, S., Li, J., Fornoni, A., Goes, N., Sageshima, J., et al. (2011). Circulating urokinase receptor as a cause of focal segmental glomerulosclerosis. Nat. Med. 17, 952-960. doi: 10.1038/nm.2411

Yang, Q., Ren, G. L., Wei, B., Jin, J., Huang, X. R., Shao, W., et al. (2019). Conditional knockout of TGF-betaRII/Smad2 signals protects against acute renal injury by alleviating cell necroptosis, apoptosis and inflammation. Theranostics 9, 8277-8293. doi: 10.7150/thno.35686

You, Y., Borgmann, K., Edara, V. V., Stacy, S., Ghorpade, A., and Ikezu, T. (2020). Activated human astrocyte-derived extracellular vesicles modulate neuronal uptake, differentiation and firing. J. Extracell. Vesicles 9:1706801. doi: 10.1080/20013078.2019.1706801

Yuan, D., Zhao, Y., Banks, W. A., Bullock, K. M., Haney, M., Batrakova, E., et al. (2017). Macrophage exosomes as natural nanocarriers for protein delivery to inflamed brain. Biomaterials 142, 1-12. doi: 10.1016/j. biomaterials.2017.07.011

Zhang, W., Zhang, X., Zhang, L., Xu, D., Cheng, N., Tang, Y., et al. (2020). Astrocytes increase exosomal secretion of oligodendrocyte precursor cells to promote their proliferation via integrin beta4-mediated cell adhesion. Biochem. Biophys. Res. Commun. 526, 341-348. doi: 10.1016/j.bbrc. 2020.03.092

Zhu, H., Liao, J., Zhou, X., Hong, X., Song, D., Hou, F. F., et al. (2020). Tenascin-C promotes acute kidney injury to chronic kidney disease progression by impairing tubular integrity via alphavbeta6 integrin signaling. Kidney Int. 97, 1017-1031. doi: 10.1016/j.kint.2020.01.026

Zovein, A. C., Luque, A., Turlo, K. A., Hofmann, J. J., Yee, K. M., and Becker, M. S., et al. (2010). Betal integrin establishes endothelial cell polarity and arteriolar lumen formation via a Par3-dependent mechanism. Dev. Cell 18, 39-51. doi: 10.1016/j.devcel.2009.12.006

Conflict of Interest: The authors declare that the research was conducted in the absence of any commercial or financial relationships that could be construed as a potential conflict of interest.

Copyright (C) 2021 Shen, Zhong, Tang, Wang, Jing, Liu and Lv. This is an open-access article distributed under the terms of the Creative Commons Attribution License (CC BY). The use, distribution or reproduction in other forums is permitted, provided the original author(s) and the copyright owner(s) are credited and that the original publication in this journal is cited, in accordance with accepted academic practice. No use, distribution or reproduction is permitted which does not comply with these terms. 